\title{
The effects of effort toward comprehension on recall
}

\author{
PAMELA M. AUBLE and JEFFERY J. FRANKS \\ Vanderbilt University, Nashville, Tennessee 37203
}

\begin{abstract}
Four experiments examined free recall of generally incomprehensible sentences with appropriate cues that made the sentences comprehensible. A distinction is made between processes involved in an effort toward comprehension and elaboration processes that occur following comprehension. It was found that providing additional time for effort toward comprehension enhanced recall, while providing additional time for elaboration following comprehension did not enhance recall. It was also shown that the effects of effort toward comprehension were contingent upon eventual understanding of the sentence.
\end{abstract}

The present experiments were designed to extend previous research on the effect of comprehending a stimulus and to clarify the effects of elaboration of a stimulus in relation to comprehension. The question to be addressed is whether the effects of elaboration are contingent on where or how comprehension occurs.

Several studies have shown the importance for later recall of comprehending a stimulus. For example, Marks and Miller (1964) and Schulman (1974) showed that meaningful stimuli are better recalled than nonsensical stimuli. It was also demonstrated by Bransford and Johnson (1972) and Bransford and McCarrell (1974) that even potentially meaningful stimuli are better recalled in the presence of appropriate contextual information. Johnson, Doll, Bransford, and Lapinski (1974) showed that generally incomprehensible sentences were recalled less well if subjects received an inappropriate context or no context than if they received appropriate contexts.

The notions of elaboration or deeper processing of a stimulus have also been stressed as important for facilitating later recall (Craik \& Lockhart, 1972; Lockhart, Craik, \& Jacoby, 1975). For instance, Epstein, Phillips, and Johnson (1975) found that finding similarities between words with different meanings or differences between words with similar meanings resulted in better recall than when subjects found similarities between similar words or differences between different words. This could be interpreted as showing that greater elaboration and thus better recall resulted when the subjects participated in a more difficult task.

Similarly, in a study by Stoff and Eagle (1971) it was demonstrated that slower word presentation rates facilitated recall if subjects used more elaborative organizational learning strategies than if subjects used word rehearsal strategies. However, the results of Experiment 7 in Craik and Tulving (1975) suggest that elaborative semantic processing might not be helpful for later recall if comprehension of the stimulus is not achieved. They found that more elaborated sentence frames facilitated later recall of the target word only if the target word fit meaningfully into the sentence frame. Thus it seems that comprehension is important for elaboration of a stimulus to be effective in facilitating recall. The purpose of the present work was to more precisely articulate the nature of the relationship between comprehension and elaboration as this relationship affects memory. More particularly, in the studies below we distinguish between elaborative processing that involves an attempt to comprehend a stimulus and processing that involves elaboration of an already comprehended stimulus. The former type of processing we will term "effort toward comprehension," while we will continue to refer to the latter as "elaboration." It is unclear from prior research whether memory enhancement attributable to elaboration (e.g., Craik \& Tulving, 1975, Experiment 7) can be differentially attributed to one as opposed to the other of these two kinds of elaborative processes. The first experiment was conducted to clarify this question.

In the first experiment, and in all the following experiments, subjects were presented with generally incomprehensible sentences such as those used by McCarrell, Bransford, and Johnson (cited in Bransford \& McCarrell, 1974). For example, "The street was full of holes because the turning stopped," is not easily understandable without the cue "cement mixer." In the first experiment, the incomprehensible sentences were presented with appropriate cues following the sentences at one of three different time intervals. For one-third of the items, the sentences were presented with the cue following immediately after the sentence and with a 5 -sec interval after the cue (5-after). The 5-after condition represents instances where subjects are given time for elaboration after comprehension is achieved. One-third of the sentences were presented with a 5 -sec interval after each sentence but before the cue (5-before) to allow time for the type of elaboration we have termed effort toward comprehension. One-third of the sentences were presented with no 
interval before or after the cue (no-time). It was hypothesized that extra time to process a sentence (as in the 5-before and 5-after conditions) would result in higher recall rates than when no time is presented, as in the no-time condition (Stoff \& Eagle, 1971; Johnson, et al., 1974). If elaboration is more effective when it occurs after understanding is achieved, it would be expected that recall rates for the 5 -after condition would be higher than for the 5 -before condition. If elaboration is more effective when it involves an effort toward comprehension of the stimulus, it would be expected that recall rates for the 5-before condition would exceed those in the 5 -after condition.

\section{EXPERIMENT 1}

\section{Method}

Subjects. The subjects were 20 introductory psychology students at Vanderbilt University, divided into two groups of seven subjects each and one group of six subjects. They received course credit for participation in the experiment.

Materials and Procedure. A list of 21 generally incomprehensible sentences and their appropriate cues were used, similar to those used by McCarrell et al. (cited in Bransford \& McCarrell, 1974). An example sentence is "The notes were sour because the seam split," with the appropriate cue being "bagpipe."

During acquisition, subjects were read the sentences, each followed by its appropriate cue in an intentional learning task. Seven sentences were presented with an interval of $5 \mathrm{sec}$ before presentation of their cues (5-before), seven sentences were presented with an interval of $5 \mathrm{sec}$ after the cue (5-after), and seven sentences were presented with no interval either before or after the cue (no-time). These three types of sentences were randomly intermixed during presentation. Three different acquisition lists were formed, and the three different interval conditions were counterbalanced over sentences such that across lists, each sentence appeard with each type of interval.

After each sentence cue and the appropriate time interval were presented, the experimenter said "Mark," and the subjects marked whether they understood the sentence or not. The subjects were given $3 \mathrm{sec}$ to rate the sentence before the next sentence was presented. After all the sentences were read, and a short pause, the subjects were given $9 \mathrm{~min}$ for free recall of as many of the sentences and their cues as possible. Partial recall was encouraged.

\section{Results and Discussion}

The number of "no" and "yes" responses marked during acquisition were scored. Subjects indicated that they understood $82.1 \%$ of the sentences in the no-time condition, $82.8 \%$ of the sentences in the 5 -after condition, and $85.0 \%$ in the 5-before condition. Thus, the sentences were generally well understood by the subjects after the cues were presented.

The recall results were scored on both the number of words remembered correctly and the number of sentences or close paraphrases recalled by each subject. The results of these two types of scoring were very similar, so only the sentence scoring will be reported.

Recall was highest for the 5-before condition (mean $=33.9 \%, \mathrm{SD}=.145)$ and second for the 5-after condition (mean $=23.9 \%, \mathrm{SD}=.176)$. The sentences in the no-time condition were least well recalled (mean $=18.8 \%, \mathrm{SD}=.150$ ). The scores were analyzed by a 3 by 3 mixed-design ANOVA with the three different cue intervals being a within-subjects factor and the three counter-balanced lists being a between-subjects factor. The main effect due to the different intervals was significant $[F(2,34)=4.84, p<.05]$. The effect due to lists was not significant $[F(2,17)=3.44, p>.05]$, nor was the interaction significant $[F(4,34)=4.34$, $\mathrm{p}>.05]$.

Comparison between the different interval conditions revealed that the 5 -before condition led to significantly greater recall than the no-time condition $[t(38)=3.07, p<.01]$. The difference between the 5 -after and no-time conditions was not significant $[t(38)=1.04, p>05]$. The difference between the 5 -before and 5-after was borderline significant at the .05 level $[\mathrm{t}(38)=2.03]$.

One possible interpretation of these differences could be that the slightly higher comprehension ratings $(85.0 \%)$ in the 5-before condition than in the other two conditions $(82.8 \%$ and $82.1 \%)$ led to subsequent higher recall in this condition. This interpretation would be congruent with research showing that comprehensible stimuli are remembered better than stimuli that are not understood. However, differences in comprehension ratings were very small. More importantly, in the following experiments, it was found that when subjects are given an understandable sentence, comprehension ratings always range between $80 \%$ and $88 \%$, and, within the range, higher comprehension ratings and higher recall rates were not necessarily positively correlated.

Thus, it appears that providing time for processing prior to comprehension of a sentence (5-before) is more effective in enhancing recall than either of the other conditions. This suggests that elaborative processing that involves an effort toward comprehension is an effective determinant of later remembering. While Experiment 1 indicates that time for effort toward comprehension (5-before) may be more effective than time for elaboration following comprehension (5-after) the borderline significance obtained here is equivocated by the results of Experiment 2 below. The equivocal nature of this effect may be due to the 5-after condition requiring a substantial degree of effort toward comprehension. The results of the first experiment suggest that processing involving an effort toward comprehension is an important determinant of later recall. However, further consideration of the materials in Experiment 1 also suggested that all three conditions might have involved significant degrees of effort toward comprehension. Note that cues always followed an apparently nonsensical sentence. To understand the sentence, the subject would have to integrate the cue word and the sentence in a meaningful way, and this would require some effort toward comprehension. Experiment 2 was designed to further investigate the 
effects of different degrees of effort toward comprehension. In the second experiment, a new condition was introduced that required less effort toward comprehension by the subjects. The conditions of 5-before and 5-after were again used and a new condition consisting of sentences with the cue incorporated meaningfully into the sentence was also included (within). For example, "The party was stalled because the wire straightened," with the cue "corkscrew" was changed to read "The party was stalled because the corkscrew wire straightened," thus eliminating the initial impression by subjects that the sentence is nonsensical and must be made meaningful by integrating the sentence with the cue following it. A 5-sec interval was presented after each sentence in this condition. Because the within condition presented subjects with sentences in which the cue information was already meaningfully integrated, it was expected that less effort toward comprehension would be put forth by subjects and that recall rates in this condition would be lower than in the 5-after and 5-before conditions. In accordance with the results of Experiment 1, it was expected that recall rates in the 5-before condition will exceed those in the 5 -after condition.

\section{EXPERIMENT 2}

\begin{abstract}
Method
Subjects. The subjects were 24 introductory psychology students at Vanderbilt University, randomly assigned to one of three groups composed of nine, eight, and seven subjects. They received course credit for participation in the experiment.

Materials and Procedure. Subjects were read a randomized list consisting of five incomprehensible sentences with cues incorporated into the sentence (within), and 10 incomprehensible sentences with cues following each sentence in an intentional learning task. Five of the sentences with post cues were presented with an interval of $5 \mathrm{sec}$ before the cue (5-before), and five were presented with an interval of $5 \mathrm{sec}$ after the cue (5-after). The sentences with cues incorporated into them were presented with an interval of $5 \mathrm{sec}$ after the sentence. Again, three lists were formed and the conditions were counterbalanced so that each sentence appeared in each of the three different conditions.

The acquisition procedure was identical to Experiment 1 except that the scale for rating understanding was changed to include a "not sure" response choice. After the sentences were presented, the experimenter paused for $1 \mathrm{~min}$ and then asked the subjects to recall as many of the sentences as they could. Subjects were given $7 \mathrm{~min}$ for recall.
\end{abstract}

\section{Results and Discussion}

The understanding measure was scored and the number of "did not understand," "not sure," and "understood" responses were computed. As in Experiment 1 , it was found that the sentences were generally well understood after the cues were received. The comprehension ("yes" response) rate was $80.9 \%$ in the 5 -after condition, $82.5 \%$ in the 5-before condition, and $84.2 \%$ in the within condition.

The recall results were scored in terms of the number of sentences or sentence paraphrases remembered. The 5-before condition again resulted in the highest recall rates (mean $=40.4 \%, \mathrm{SD}=.261$ ); the 5 -after condition had intermediate recall rates (mean $=36.6 \%, \mathrm{SD}=.188$ ); and the sentences in the within condition were recalled least well $($ mean $=25.3 \%, \mathrm{SD}=.210)$. Scores were analyzed using a 3 by 3 mixed-design ANOVA, with the within-subjects factor being the interval and cue placement conditions and the between-subjects factor being the three lists. The main effect due to the cue interval and placement were significant $[F(2,42)=3.94$, $\mathrm{p}<.05]$; The effects due to the lists was not significant $[F(2,21)<1.0]$, nor was the interaction significant $[F(4,42)<1.0]$.

Comparisons using $t$ tests were again used to further analyze the data. Recall in the 5-after condition was significantly higher than the within condition $[\mathrm{t}(42)=$ $2.04, \mathrm{p}<.05$ ]. Thus, it seems that putting forth effort toward comprehension by actively relating the momentarily incomprehensible sentence with its post cue is an important variable affecting recall. Significant differences were also obtained between the 5-before and within conditions $[\mathrm{t}(42)=2.71, \mathrm{p}<.01]$, further supporting this interpretation.

Although recall for the 5-before condition was slightly higher than for the 5-after condition, this difference was not significant $[\mathrm{t}(42)=.67, \mathrm{p}>.05]$, thus equivocating the borderline significance found for this difference in Experiment 1. Therefore, it seems that whether the extra time to make sense of the sentences and elaborate them is given before or after the cue is not as important as having sentences and cues that must be actively integrated by subjects in their effort toward comprehension. The higher recall exhibited in the 5-before condition than in the 5-after condition (in conjunction with the difference found in Experiment 1) suggests that the effort toward comprehension of a sentence could be slightly more effective for later recall if the cue is not immediately presented. Although, again, the mere presence of an incomprehensible sentence that must be connected with the cue seems to be the most important factor in later recall under present conditions. In relation to the discussion of Experiment 1, note that the comprehension ratings for the three conditions in the present study were again very similar. The slight differences that did occur, with the within condition being the highest, were not positively correlated with later recall, in which the within condition exhibited the lowest recall.

The within condition in Experiment 2 was chosen to minimize subjects' efforts toward comprehension. In this case, the 5 -sec post sentence interval could be expected to provide relatively more time for elaborative processing following comprehension. The lesser recall in the within condition indicates that, to the extent such elaboration following comprehension is occurring, the effects are less potent in enhancing recall that the 
processing involved in efforts toward comprehension. Experiment 3 was designed to assess the effects of elaboration following comprehension under conditions where effort toward comprehension was minimized. In this experiment, only the sentences with the cues meaningfully embedded in them were used. These sentences were presented with either $5 \mathrm{sec}$ following the sentence (within-5), or no interval following the sentence (within-0). If more time after a sentence is effective in increasing elaboration and recall without effort toward comprehension, then recall rates in the within-5 condition should be higher than those in the within- 0 condition.

\section{EXPERIMENT 3}

\section{Method}

Subjects. The subjects were 17 introductory psychology students at Vanderbilt University, randomly assigned to one group of nine subjects and one group of eight subjects. They received course credit for participation in the experiment.

Materials and Procedure. Subjects were read a list of 16 incomprehensible sentences with the cues meaningfully embedded in them in an intentional learning task. Eight sentences were presented with an interval of $5 \mathrm{sec}$ following each one (within-5) and eight sentences were presented with no interval (within-0). These two types of sentences were randomly intermixed during presentation. Two different acquisition lists wete formed and the two interval conditions were counterbalanced across lists so that each sentence appeared in each condition. The experimental procedure was identical to that of Experiment 2 .

\section{Results and Discussion}

Recall results were scored in terms of the number of sentences or close paraphrases remembered. Rates of recall were very similar for the within-5 condition (mean $=31.1 \%, \mathrm{SD}=.129)$, and the within -0 condition (mean $=30.3 \%, \mathrm{SD}=.106)$. The data were analyzed by a 2 by 2 mixed-design ANOVA with the within-subjects factor being the 0 - or 5 -sec intervals and lists as the between-subjects factor. The main effect due to the different interval conditions was not significant $[\mathrm{F}(1,15)<1.0]$. The effect due to the different lists $[F(1,15)=2.49, p<.05]$ was also not significant, nor was the interaction between the two factors significant $[\mathrm{F}(1,15)<1.0]$.

Therefore, it seems that under the present experimental conditions, providing additional time after relatively easily comprehended sentences does not lead to additional elaboration following comprehension that enhances later recall. This finding is somewhat at odds with the literature concerend with affects of additional time on the memory for word lists, where one usually finds that greater time for semantic or elaborative rehearsal of words enhances recall (e.g., Stoff \& Eagle, 1971). However, a major difference between the present study and the previous work is that the present experiment utilizes sentential rather than word stimuli. Unlike individual words, these sentences already consist of relatively elaborate semantic structures to be comprehended. It may well be that under such conditions, where the stimuli to be comprehended are already relatively complex, subjects may not engage in additional elaborative processing. Rather, during additional provided time they may just do nothing or possibly engage in a maintenance type of rehearsal that might not be expected to enhance recall (Craik \& Watkins, 1973; Woodward, Bjork, \& Jongeward, 1973).

Alternatively, one might argue that studies using individual words might be construed as situations that provide subjects with relatively impoverished semantic inputs. That is, presentation of individual words might be somehow analogous to presentation of the present sentential stimuli without the cues needed for effective comprehension. From this view, additional time for elaborative processing might be considered to provide additional time for the subjects to engage in effort toward comprehension that involves integrating the word into a more complex semantic structure (e.g., Stoff \& Eagle, 1971). To the extent this latter argument holds, the results of the present work, indicating the relative importance of efforts toward comprehension for enhancing recall, could be considered to be compatible with the effects found for individual word stimuli. Further work is needed to clarify the effects of additional time for sentences as compared with words. However, the complexity of the questions involved will necessitate a whole line of additional experiements; thus, for present purposes it seems sufficient to merely note that we have replicated Experiment 3 with an additional 15 subjects and a somewhat different set of sentences. In this replication, once again no difference was found between the within- 5 and within- 0 conditions $[$ within -5 mean $=25.8 \%$; within -0 mean $=$ $24.3 \% ; \mathrm{t}(14)=.68]$.

The results of the comprehension measure again indicated that the majority of the sentences were understood by the subjects (mean $=84.6 \%$ for the within -0 condition; mean $=81.6 \%$ for the within -5 condition). It was also noted that when a sentence was reported to have been understood, that sentence was more often recalled later than when a subject reported not understanding the sentence. Overall, of the sentences they reported understanding, subjects recalled $33.3 \%$ and $32.2 \%$ of the sentences in the within-5 and within-0 conditions, respectively. The recall rates were only $18.4 \%$ and $15.4 \%$, respectively, for sentences subjects reported not understanding or were unsure if they understood. Similar patterns of recall conditional on reported comprehension were found in Experiments 1 and 2. Collapsing across all the conditions in these previous experiments, it was found that, overall, subjects recall $33.4 \%$ of the sentences they reported understand. ing and only $19.1 \%$ of the sentences they reported not understanding or were unsure if they understood.

Bransford and Johnson (1972), Bransford and 
McCarrell (1974), and Craik and Tulving (1975) also demonstrated the importance of comprehending a stimulus in facilitating later recall. Based on this previous work, it might be expected that the effectiveness of efforts toward comprehension in enhnacing recall would be contingent upon eventual comprehension of the stimuli. The experiments above demonstrate the effectiveness of efforts toward comprehension. However, the materials and procedures of these experiments were designed such that, in all conditions, subjects comprehended most of the sentences. Experiment 4 was designed to examine the effects of effort toward comprehension under conditions where subjects did not eventually comprehend the sentences. A within-subjects design was used with four conditions. In two conditions, incomprehensible sentences were presented without the cue. In one of these conditions, subjects received $5 \mathrm{sec}$ after the sentence to attempt to understand it (no-cue-5) and in the other condition no time was given (no-cue-0). If effort toward comprehension per se is the most important variable in later recall, the 5 -sec interval condition should be significantly higher than the no-interval condition. If, however, the effectiveness of effort toward comprehension is contingent upon eventual comprehension of the sentence, these conditions should not be significantly different.

In the other two conditions, incomprehensible sentences were presented with cues following the sentences as in Experiment 1. One of these conditions was presented with a 5 -sec precue interval (5-before), and the other was presented with no interval (no-time). If effort toward comprehension is most effective when understanding is eventually achieved, then the sentences with cues should be better recalled than those without cues. Also, in replication of the results of Experiment 1, the 5-before condition was expected to result in greater retention than the no-time condition.

\section{EXPERIMENT 4}

\section{Method}

Subjects. The subjects were 23 introductory psychology students at Vanderbilt University randomly assigned to one group of five subjects and three groups of six subjects each. They received course credit for participation in the experiment.

Materials and Procedure. Subjects were read a list of 16 incomprehensible sentences in an intentional learning task. Four sentences were presented with no cue and a 5-sec interval after the sentence (no-cue-5 condition), four sentences were presented with no cue and no interval (no-cue- 0 condition), four sentences were presented with a cue following the sentence and a 5 -sec precue interval (5-before), and four sentences were presented with a post cue but no interval (no-time). These four types of sentences were radomly intermixed during presentation. Four different acquisition lists were formed and the four different interval and cue conditions were counterbalanced over sentences such that across lists, each sentence appeared in each type of interval. The experimental procedure was identical to Experiment 2.

\section{Results and Discussion}

Recall in the 5-before condition was again highest (mean $=48.0 \%, \mathrm{SD}=.271)$. The no-time condition was next (mean $=32.3 \%, \mathrm{SD}=.201$ ). The subjects recalled sentences in the no-cue conditions least well. The recall results for the no-cue-5 condition (mean $=20.8 \%$ $\mathrm{SD}=.175)$ were almost identical with the no-cue-0 condition (mean $=19.2 \%, \mathrm{SD}=.127$ ). The scores were analyzed by a 4 (between-subjects factor of counterbalanced lists) by 2 (within-subjects factor of cue vs. no cue) by 2 (within-subjects factor of $0-$ vs. 5 -sec interval) ANOVA. A significant mean effect for the presence or absence of a cue was obtained $[F(1,19)=$ $31.0, \mathrm{p}<.001]$. A significant main effect was also obtained due to interval conditions $[F(1,19)=5.72$, $\mathrm{p}<.05]$. The interaction between the cue and interval conditions was significant $[F(3,19)=4.24, p=.051]$. The effect of the counterbalanced lists was not significant $[F(3,19)<1.0]$, nor were any of the other interactions significant.

To analyze these results further, individual t-test comparisons were again examined. First, cue and no-cue groups with equal intervals were compared. Differences were significant between the 5-before and no-cue-5 conditions $[\mathrm{t}(57)=5.35, \mathrm{p}<.001]$ and between the no-time and no-cue- 0 conditions $[\mathrm{t}(57)=2.50, \mathrm{p}<.05]$. This supports the hypothesis that later recall is facilitated if a person eventually understands the sentence.

Comparing the 5-before and no-time conditions resulted in significant difference $[t(57)=3.17, \mathrm{p}<.01]$. This replicates the findings of Experiment 1 and again supports the position that if a sentence is eventually understood, extra time spent in an effort toward comprehension is effective in facilitating later recall. However, the comparison between the no-cue- 5 and no-cue- 0 conditions was not significant $[t(57)=.317]$. This lack of difference, combined with the significant difference between the 5-before and no-time conditions, implies that the effectiveness of effort toward comprehension is contingent upon eventual understanding of the sentences. In the no-cue conditions in which comprehension of the sentences was minimized, providing subjects with an additional $5 \mathrm{sec}$ to engage in efforts toward comprehension did not result in facilitated recall. In the cue conditions, in which comprehension was high, the added $5 \mathrm{sec}$ was effective in enhnacing recall.

The scores of the understanding measure from the no-cue conditions indicated that the no-cue manipulation was effective in decreasing understanding of the sentences. Without the cue, understanding was not achieved the majority of the time. Subjects reported not understanding the sentence or not being sure if they understood it $70.6 \%$ of the time in the no-cue- 0 condition and $63.0 \%$ of the time in the no-cue-5 condition, compared with only $16.3 \%$ and $12.0 \%$ responses of not understanding or being unsure in the 5-before and no-time conditions, respectively. It was also observed that when subjects reported understanding sentences in the no-cue conditions, they recalled $25.9 \%$ and $29.4 \%$ of these sentences in the no-cue- 0 and no-cue-5 conditions, respectively. When no-cue sentences were 
reported as not understood, the recall rates were lower, only $16.9 \%$ for no-cue-0 and $15.5 \%$ for no-cue-5. Likewise, in the no-time and 5-before conditions, subjects recalled $33.3 \%$ and $51.9 \%$, respectively, of the sentences they reported understanding and recalled only $27.3 \%$ and $26.7 \%$ of the sentences they did not understand. These results further support the position that eventual comprehension of the sentences is important for later recall.

\section{SUMMARY}

The present experiments provide some initial clarification of the relation between comprehension and elaboration as these affect recall of sentential material. In general, the results indicate the potential importance of distinguishing between elaborative processing that is involved in effort toward comprehension and elaboration that might occur following comprehension of a sentence. The results of Experiment 1 indicate that under conditions where subjects are presumably actively involved in efforts to understand sentences, providing additional time for this effort toward comprehension facilitates later recall. The findings of Experiment 2 demonstrate that if time per sentence is held constant, conditions that minimize efforts toward comprehension result in lower recall than conditions that promote greater efforts toward comprehension. Experiment 3 indicates that under conditions that minimize efforts toward comprehension. providing additional time for elaboration following comprehension does not enhance later recall. The results of Experiment 4 indicate that the recall enhancement attributable to efforts toward comprehension is contingent upon eventual comprehension of the sentences. If sentences are not comprehended, providing extra time for efforts toward comprehension does not lead to increases in recall performance.

\section{REFERENCES}

Bransford, J. D., \& Johnson, M. K. Contextual prerequisites for understanding: Some investigations of comprehension and recall. Journal of Verbal Learning and Verbal Behavior. 1972. 11, 717-720.

Bransford, J. D. \& \& MCCarrell, N. S. A sketch of a cognitive approach to comprehension. In W. Weimer \& D. Palermo (Eds.), Cognition and the symbolic processes. Hillsdale, N.J: Lawrence Erlbaum, 1974.

CRAIK. F. I. M., \& LockharT, R. S. Levels of processing: A framework for memory research. Journal of Verbal Learning and Verbal Behavior. 1972, 11, 671-684.

Craik, F. I. M., \& Tulving. E. Depth of processing and the retention of words in episodic memory. Journal of Experimental Psychology: General, 1975, 104, 268-294.

Craik. F. I. M., \& WATKINS, M. J. The role of rehearsal in short-term memory. Joumal of Verbal Learning and Verbal Behavior. 1973, 12, 599-607.

Epstein, M. L., Phillips, W. D., \& Johnson, S. S. Recall of related and unrelated word pairs as a function of processing level. Joumal of Experimental Psychology: Human Learning and Memon, 1975, 104, 149-152.

Johnson, M. K., Doll, T. J., BRansford, J. D., \& LAPINSKI, R. Context effects in sentence memory. Journal of Experimental Psychology, 1974, 103, 358-360.

Lockhart, R. S., Craik, F. I. M., \& Jacoby, L. L. Depth of processing in recognition and recall: Some aspects in a general memory system. In J. Brown (Ed.), Recognition and recall. London: Wiley, 1975.

Marks, L. E., \& Miller, G. A. The role of semantic and syntactic constraints in the memorization of English sentences. Journal of Verbal Learning and Verbal Behavior, $1964,3,1-5$.

Schulman, A. I. Memory for words recently classified. Memory \& Cognition, 1974, 2. 47-52.

StofF, M.. \& EAGLE, M. N. The relationship among reported strategies, presentation rate, and verbal ability and their effects on free recall learning. Journal of Experimental Psychology, 1971, 87, 423-428.

WoOdward, A. E., JR., Biork, R. A., \& Jongeward, R. H., $J_{R}$. Recall and recognition as a function of primary rehears. al. Journal of Verbal Learning and Verbal Behavior, 1973, 12. 608-617.

(Received for publication July 20, 1977; accepted November 7, 1977.) 